\title{
Evolución clínica de pacientes hospitalizados por dengue en una institución de salud de Bucaramanga, Colombia
}

\author{
Andrés Leonardo González, Ruth Aralí Martínez, Luis Ángel Villar \\ Grupo de Epidemiología Clínica, Centro de Investigaciones Epidemiológicas, Universidad Industrial de \\ Santander, Bucaramanga, Santander \\ Trabajo realizado en la Clínica Chicamocha S.A. \\ Introducción. El dengue hemorrágico abarca todas las áreas tropicales y subtropicales del \\ planeta, y causa medio millón de hospitalizaciones al año en el mundo. Se ha descrito que \\ esta enfermedad afecta a un grupo cada vez mayor de adolescentes y adultos jóvenes. \\ Objetivo. Describir las características clínicas de la población, tanto adulta como pediátrica, \\ hospitalizada por dengue durante un periodo endémico y determinar los factores de riesgo \\ asociados a mal pronóstico. \\ Materiales y métodos. Cohorte retrospectiva de pacientes hospitalizados por dengue en los \\ años 2006 y 2007 en la Clínica Chicamocha. \\ Resultados. Se evaluaron 328 pacientes, 165 mujeres y 163 hombres, con mediana de edad \\ de 25 años. Se encontraron 116 casos de dengue hemorrágico, de los cuales, 113 eran de \\ grado II. De los 212 pacientes con dengue clásico, 156 presentaron extravasación, sangrado \\ o trombocitopenia. El 82,4\% tuvieron serología positiva. Los pacientes con dengue hemorrágico \\ eran más jóvenes $(20,1$ contra 25,7 años, $p=0,0054)$. El número mínimo de plaquetas y el valor \\ máximo del hematocrito se alcanzaron alrededor del sexto día de enfermedad. Los menores \\ de 13 años presentaron con menor frecuencia los síntomas típicos de dengue, aunque con un \\ mayor porcentaje de ascitis, derrame y sangrado; además, tuvieron mayor riesgo de desarrollar \\ dificultad respiratoria (riesgo relativo $(R R)=3,59, I C 95 \% 1,3-9,9, p=0,014$ ) e hipotensión \\ (RR=10,77, IC95\% 5,56-20,86, $\mathrm{p}<0,001)$. \\ Conclusiones. La edad continúa siendo el factor predominante en la gravedad intrahospitalaria \\ del dengue. Independientemente de ésta, un grupo de signos, síntomas y hallazgos de \\ laboratorio al ingreso permite predecir la aparición de complicaciones.
}

Palabras clave: fiebre dengue hemorrágica, hospitalización, pronóstico, adulto, niño, estudios de cohortes.

\section{Clinical evolution of dengue in hospitalized patients}

Introduction. Dengue hemorrhagic fever has extended to every tropical and subtropical area of the world, resulting in a half million hospitalizations every year. This disease appears to affect increasing numbers of adolescents and young adults.

Objective. The clinical characteristics were described for adult and pediatric dengue inpatients to establish risk factors associated with bad prognosis.

Materials and methods. A cohort of dengue inpatients of years 2006 and 2007 was evaluated retrospectively at "Clínica Chicamocha", a high level hospital in Bucaramanga, Colombia.

Results. Of 328 patients evaluated, 165 were female and 163 were male with a median age of 25 years. Dengue hemorrhagic fever was diagnosed in 116 patients, of which 113 were classified grade II. Of the 212 patients with dengue fever, 156 developed signs of plasma leakage, bleeding or thrombocytopenia. A positive serology was indicated in $82.4 \%$ of the patients. Inpatients with dengue hemorrhagic fever were younger (20.1 vs. 25.7 years, $p<0.0054)$. Both lowest level of platelets and highest hematocrit were reached at the sixth day of illness. Children presented the typical symptoms of dengue less frequently, but demonstrated a greater 
proportion of ascites, pleural effusion and bleeding, and a higher risk of developing respiratory distress $(R R=3.59,95 \% C l$ 1.3-9.9, $p<0.014)$ and hypotension $(R R=10.77,95 \% \mathrm{Cl} 5.56-20.86$, $\mathrm{p}<0.001$ ).

Conclusions. Age was the most determinant factor of severity in dengue inpatients. In addition, a combination of particular symptoms and laboratory data at the day of admission may predict the development of complications.

Key words: Dengue hemorrhagic fever, hospitalization, prognosis, adult, child, adolescent, cohort studies.

Desde su primera descripción en 1950, el dengue hemorrágico ha tenido un crecimiento constante hasta abarcar todas las áreas tropicales y subtropicales del planeta, con 50 a 100 millones de casos anuales y medio millón de hospitalizaciones, de acuerdo con la Organización Mundial de la Salud (OMS) (1). Esta infección de transmisión por vectores es causada por cuatro serotipos del virus dengue, $1,2,3$ y 4 , cuya infección produce un amplio espectro clínico que va desde el leve pero incapacitante dengue clásico, hasta el dengue hemorrágico y el síndrome de choque por dengue, responsables de más de 24.000 muertes al año. Factores tales como la virulencia de los serotipos, la producción de anticuerpos no neutralizantes, la cascada inflamatoria mediada por citocinas y la activación del complemento, se consideran responsables de la fuga plasmática, la trombocitopenia y el sangrado causados por la enfermedad (2).

En el 2007, América reportó a la Organización Panamericana de la Salud (OPS) 26.413 casos de dengue hemorrágico, de los cuales, 4.665 correspondían a Colombia. Asimismo, nuestro país notificó epidemias en 1995, 1998 y 2001 (3-5). El departamento de Santander muestra la mayor actividad de la enfermedad en el país, con $48,6 \%$ y $28,2 \%$ de los casos registrados en 2006 y 2007 , respectivamente $(6,7)$.

Sin vacuna ni terapia antiviral disponibles, el abordaje del dengue se basa en el control del

\footnotetext{
Correspondencia:

Andrés Leonardo González, Centro de Investigaciones Epidemiológicas, Facultad de Salud, Universidad Industrial de Santander, Carrera 32 № 29-31, oficina 304, Bucaramanga, Colombia.

Teléfono: (7) 634 4000, extensión 3205; fax: (7) 6345781 andreslgonzalezr@gmail.com

Recibido: 01/04/08; aceptado:03/09/08
}

vector, el diagnóstico oportuno y un tratamiento que comprende manejo sintomático, soporte hídrico y observación, lo cual puede reducir la mortalidad a menos de $1 \%$, a la vez que se racionan los recursos hospitalarios (8). Nuestro sistema de salud promueve la hospitalización oportuna ante la presencia de signos de alarma indicativos de complicación (9) y la endemia actual se maneja exitosamente pese a las dificultades encontradas en el diagnóstico clínico (10).

A medida que el patrón local de enfermedad se parece más al de los países asiáticos, donde el dengue es hiperendémico y muestra gran variabilidad clínica entre poblaciones y periodos (11-18), se hace necesario recolectar nueva evidencia que permita generar recomendaciones específicas para nuestra población. Un punto de partida adecuado para ello consiste en el análisis de los pacientes hospitalizados, que representan la punta del iceberg en términos de gravedad. En Colombia se han realizado cinco estudios de este tipo: el primero, en Cali, con 99 adultos y niños, en el cual se diferenció clínicamente el dengue hemorrágico de otras formas de la enfermedad y se comprobó el papel del dolor abdominal como signo de alarma (19); el segundo, en Neiva, con 246 niños, que mostró una alta prevalencia de síntomas abdominales y choque, así como una elevada mortalidad en menores de dos años (20); el tercero, en Bucaramanga, con 763 niños, el cual confirmó en nuestro medio la dificultad para clasificar los pacientes según la definición de la OMS y una elevada frecuencia de las formas graves en los pacientes mayores de cinco años (21); y el cuarto, en Apartadó, con 45 adultos y niños, que mostró una proporción importante del llamado "dengue con manifestaciones hemorrágicas", un mayor número de casos entre los 15 y los 24 años y una elevada morbilidad asociada (22). El trabajo 
más reciente se llevó a cabo en Neiva, con 105 pacientes pediátricos, en el cual se destacó la importante gravedad descrita y la asociación entre las diferentes complicaciones (23).

Varios estudios tanto del sudeste asiático como de Latinoamérica confirman que la tendencia del dengue hemorrágico a afectar un grupo de mayor edad continúa, comprometiendo particularmente a adolescentes y adultos jóvenes (24). El objetivo de este trabajo es describir las características clínicas de la población hospitalizada por dengue durante un periodo endémico en una institución especializada de Bucaramanga y determinar los factores de riesgo asociados a mal pronóstico en población mixta.

\section{Materiales y métodos}

Se trata de un estudio de tipo cohorte retrospectiva de la Clínica Chicamocha, una institución privada de cuarto nivel que atiende a 300.000 usuarios del régimen contributivo en Bucaramanga y el área metropolitana. El servicio de hospitalización, con 78 camas, atendió a 5.032 y 7.231 pacientes en 2006 y 2007 , respectivamente.

Se incluyeron pacientes hospitalizados con diagnóstico clínico de ingreso de dengue clásico (A90X) o hemorrágico (A91X) desde el primero de enero de 2006 hasta el 31 de diciembre de 2007, según la décima versión de la clasificación estadística internacional de enfermedades y otros problemas de salud.

Se extrajo la información de las historias clínicas desde el primer contacto del paciente con la institución hasta el día del egreso, utilizando un instrumento diseñado para tal fin. Los resultados de las pruebas serológicas se obtuvieron con autorización de la Secretaría de Salud Departamental a partir de los registros de vigilancia epidemiológica.

Se evaluaron los siguientes desenlaces hospitalarios: hipotensión considerada como presión arterial media inferior a $60 \mathrm{~mm} \mathrm{Hg}$ en los mayores de 18 años o inferior al percentil 5 para la edad en niños (25); dengue hemorrágico de acuerdo con la definición de la OMS (26-28); ingreso a la unidad de cuidados intensivos; sangrado gastrointestinal evidenciado o referido; dificultad respiratoria (según criterio clínico) y extravasación plasmática, definida como ascitis, derrame pleural (evidenciados clínicamente o mediante imágenes diagnósticas) o hemoconcentración de más del $20 \%$ respecto a un hematocrito basal o posterior a hidratación endovenosa. Se consideraron como factores de riesgo la edad y el estado clínico al ingreso (síntomas referidos, presencia de signos de alarma, alteración de los signos vitales, hallazgos de laboratorio) y, como variables de confusión, el sexo y el día de enfermedad al momento de la hospitalización.

Se expresaron las variables categóricas como porcentajes de frecuencia y las variables continuas como medianas y rangos. Las variables de interés entre grupos de estudio se compararon empleando la prueba exacta de Fisher de dos colas para variables categóricas y la prueba de igualdad de Kruskal-Wallis para variables continuas. Se calcularon los riesgos relativos (RR) con intervalo de confianza de 95\% (IC95\%) para los factores de riesgo asociados a los desenlaces que, luego, se ajustaron mediante regresión binomial o logística. Se utilizó el programa Stata, versión 9 (StataCorp, 2005) para el análisis de los datos.

El estudio fue aprobado por el Comité de Ética de la Dirección de Investigaciones de la Facultad de Salud de la Universidad Industrial de Santander.

\section{Resultados}

De 351 pacientes que cumplían los criterios de inclusión, se excluyeron 23 porque la historia clínica no estaba disponible o por descartarse el diagnóstico de dengue durante la estancia. Además, 8 pacientes se eliminaron del análisis por falta de datos en relación con el tiempo de evolución de la enfermedad.

Se obtuvo información de 328 pacientes, 50,3\% del sexo femenino. La edad osciló entre 3 meses y 80 años, con una mediana de 25 años. El 25\% de los pacientes eran menores de 13 años. La diferencia de edad por sexo no fue significativa. Se remitieron 216 pacientes de otras instituciones, mientras que 112 consultaron directamente a urgencias. Se encontró un patrón estacional en los casos, con una mayor frecuencia de hospitalizaciones entre mayo y agosto $(48,8 \%)$. 
Se obtuvo información sobre la serología de 193 pacientes, de los cuales, $82,4 \%$ resultaron positivos y $17,6 \%$ negativos para inmunoglobulina $\mathrm{M}$ ( $\operatorname{lgM})$.

\section{Consulta inicial e ingreso}

En el momento de la consulta inicial, el tiempo de evolución de los síntomas fue de 120 horas (rango: 5 a 196), 26,3\% de los pacientes se encontraba febril y $13,9 \%$ presentaba hipotensión. En el cuadro 1 se resumen los hallazgos más comunes de la anamnesis y el examen físico. Cien pacientes $(30,5 \%)$ manifestaron un sangrado en su enfermedad actual y en 120 (36,6\%) éste se evidenció en el examen físico. La hemorragia más común fue epistaxis (31\%), seguida por petequias $(14,29 \%)$, sangrado vaginal sin especificar $(14,29 \%)$, gingivorragia $(12,7 \%)$ y hematemesis $(5,6 \%)$.
Se registraron los antecedentes que pudieran afectar el comportamiento del dengue o modificar su manejo. En la consulta inicial 45 pacientes $(13,7 \%)$ manifestaron presentar una o más de estas condiciones; las más frecuentes fueron hipertensión en 10 pacientes y asma en 5 .

Sesenta y dos pacientes asistieron a control ambulatorio antes de ser hospitalizados. El día del ingreso, 50,2\% de los pacientes presentaba, al menos, un sangrado; $20,1 \%$, fiebre; $14,2 \%$, hipotensión; $10,8 \%$, taquicardia, y $25,2 \%$, taquipnea, sin diferencias por edad excepto por la hipotensión, que para todos los días de seguimiento fue mayor en los menores de 18 años (al ingreso $46,3 \%$ contra $2,3 \%$ en adultos, $\mathrm{p}<0,001)$.

Cuadro 1. Frecuencia de síntomas referidos y hallazgos al examen físico en la primera valoración.*

\begin{tabular}{|c|c|c|c|c|c|c|c|c|c|c|c|c|}
\hline \multirow{2}{*}{$\frac{\text { Síntoma }}{\text { Fiebre }}$} & \multicolumn{2}{|c|}{ Total } & \multicolumn{2}{|c|}{ Mujeres } & \multicolumn{2}{|c|}{ Hombres } & \multirow{2}{*}{$\begin{array}{c}\mathbf{p} \\
0,619\end{array}$} & \multicolumn{2}{|c|}{$\geq 13$ años } & \multicolumn{2}{|c|}{$<13$ años } & \multirow{2}{*}{$\frac{\mathbf{P}}{0,375}$} \\
\hline & 312 & $(95,12)$ & 158 & $(95,76)$ & 154 & $(94,48)$ & & 232 & $(94,31)$ & 80 & $(97,56)$ & \\
\hline Osteomialgias & 263 & $(80,18)$ & 131 & $(79,39)$ & 132 & $(80,98)$ & 0,782 & 226 & $(91,87)$ & 37 & $(45,12)$ & $<0,001$ \\
\hline Astenia & 252 & $(76,83)$ & 130 & $(78,79)$ & 122 & $(74,85)$ & 0,434 & 208 & $(84,55)$ & 44 & $(53,66)$ & $<0,001$ \\
\hline Cefalea & 228 & $(69,51)$ & 115 & $(69,7)$ & 113 & $(69,33)$ & 1 & 200 & $(81,30)$ & 28 & $(34,15)$ & $<0,001$ \\
\hline Vómito & 170 & $(51,83)$ & 100 & $(60,61)$ & 70 & $(42,94)$ & 0,002 & 118 & $(47,97)$ & 52 & $(63,41)$ & 0,016 \\
\hline Dolor abdominal & 146 & $(44,51)$ & 81 & $(49,09)$ & 65 & $(39,88)$ & 0,097 & 106 & $(43,09)$ & 40 & $(48,78)$ & 0,373 \\
\hline Náuseas & 99 & $(30,18)$ & 58 & $(35,15)$ & 41 & $(25,15)$ & 0,055 & 84 & $(34,15)$ & 15 & $(18,29)$ & 0,008 \\
\hline Escalofrío & 94 & $(28,66)$ & 42 & $(25,45)$ & 52 & $(31,9)$ & 0,223 & 88 & $(35,77)$ & 6 & $(7,32)$ & $<0,001$ \\
\hline Diarrea & 58 & $(17,68)$ & 28 & $(16,97)$ & 30 & $(18,4)$ & 0,773 & 40 & $(16,26)$ & 18 & $(21,95)$ & 0,246 \\
\hline Exantema & 50 & $(15,24)$ & 34 & $(20,61)$ & 16 & $(9,82)$ & 0,009 & 34 & $(13,82)$ & 16 & $(19,51)$ & 0,218 \\
\hline Dolor retroocular & 38 & $(11,59)$ & 24 & $(14,55)$ & 14 & $(8,59)$ & 0,12 & 35 & $(14,23)$ & 3 & $(3,66)$ & 0,009 \\
\hline Hiporexia & 38 & $(11,59)$ & 16 & $(9,7)$ & 22 & $(13,5)$ & 0,305 & 23 & $(9,35)$ & 15 & $(18,29)$ & 0,044 \\
\hline Artralgias & 35 & $(10,67)$ & 15 & $(9,09)$ & 20 & $(12,27)$ & 0,376 & 26 & $(10,57)$ & 9 & $(10,98)$ & 1 \\
\hline Prurito & 32 & $(9,76)$ & 23 & $(13,94)$ & 9 & $(5,52)$ & 0,015 & 24 & $(9,76)$ & 8 & $(9,76)$ & 1 \\
\hline Síntomas respiratorios & 20 & $(6,10)$ & 8 & $(4,85)$ & 12 & $(7,36)$ & 0,366 & 15 & $(6,10)$ & 5 & $(6,10)$ & 1 \\
\hline Síntomas autonómicos & 11 & $(3,35)$ & 5 & $(3,03)$ & 6 & $(3,68)$ & 0,77 & 10 & $(4,07)$ & 1 & $(1,22)$ & 0,303 \\
\hline \multicolumn{13}{|c|}{ Hallazgos al examen físico } \\
\hline Dolor abdominal & 167 & $(50,91)$ & 94 & $(56,97)$ & 73 & $(44,79)$ & 0,036 & 130 & $(52,85)$ & 37 & $(45,12)$ & 0,252 \\
\hline Deshidratación & 159 & $(48,48)$ & 85 & $(51,52)$ & 74 & $(45,4)$ & 0,272 & 123 & $(51,22)$ & 33 & $(40,24)$ & 0,098 \\
\hline Exantema & 137 & $(41,77)$ & 86 & $(52,12)$ & 51 & $(31,29)$ & $<0,001$ & 103 & $(41,87)$ & 34 & $(41,46)$ & 1 \\
\hline Palidez & 97 & $(29,57)$ & 48 & $(29,09)$ & 49 & $(30,06)$ & 0,904 & 68 & $(27,64)$ & 29 & $(35,37)$ & 0,209 \\
\hline Frialdad & 40 & $(12,2)$ & 25 & $(15,15)$ & 15 & $(9,2)$ & 0,128 & 32 & $(13,01)$ & 8 & $(9,76)$ & 0,56 \\
\hline Hepatomegalia & 36 & $(10,98)$ & 20 & $(12,12)$ & 16 & $(9,82)$ & 0,597 & 22 & $(8,94)$ & 14 & $(17,07)$ & 0,064 \\
\hline Eritema faríngeo & 16 & $(4,88)$ & 6 & $(3,64)$ & 10 & $(6,13)$ & 0,318 & 12 & $(4,88)$ & 4 & $(4,88)$ & 1 \\
\hline Distensión abdominal & 15 & $(4,57)$ & 6 & $(3,64)$ & 9 & $(5,52)$ & 0,442 & 7 & $(2,85)$ & 8 & $(9,76)$ & 0,015 \\
\hline Derrame & 14 & $(4,27)$ & 7 & $(4,24)$ & 7 & $(4,29)$ & 1 & 6 & $(2,44)$ & 8 & $(9,76)$ & 0,009 \\
\hline Ascitis & 11 & $(3,35)$ & 5 & $(3,03)$ & 6 & $(3,68)$ & 0,77 & 1 & $(0,41)$ & 10 & $(12,20)$ & $<0,001$ \\
\hline
\end{tabular}

* La primera valoración corresponde a la fecha de ingreso en los pacientes remitidos y a la primera consulta en los demás. $\mathrm{n}=328$

Datos mostrados como frecuencia (porcentaje) 


\section{Evolución clínica}

La estancia hospitalaria varió entre 1 y 15 días, con una mediana de 3 , sin diferencias por sexo o edad. En el cuadro 2 se muestra el comportamiento de los signos vitales según el día de enfermedad. Se encontró que 81 pacientes $(25,6 \%)$ desarrollaron hipotensión en algún momento de su evolución (74 niños Vs. 7 adultos, $p<0,001$ ) y en 14 pacientes $(4,4 \%)$ la presión de pulso cayó por debajo de 20 $\mathrm{mm} \mathrm{Hg}$ ( 3 adultos y 11 niños, $p<0,001$ ).

Las dolencias más comúnmente manifestadas fueron: prurito $(26,8 \%)$, cefalea $(16,8 \%)$, náuseas $(11,3 \%)$, diarrea $(5,5 \%)$, disnea $(4,9 \%)$ y mareo $(4,6 \%)$. Los hallazgos más frecuentes en la evolución fueron, en su orden: exantema $(57,9 \%)$, palidez $(42,7 \%)$, hepatomegalia $(23,8 \%)$, deshidratación $(21,0 \%)$, cualquier edema $(18,6 \%)$ y distensión abdominal (17,1\%). La hemorragia más común en la evolución fueron las petequias $(47 \%)$, seguidas por epistaxis $(11,6 \%)$, sangrado vaginal sin especificar $(9,2 \%)$, gingivorragia
$(6,4 \%)$, melenas $(3,7 \%)$ y hematemesis $(2,1 \%)$. En el cuadro 3 se presenta la frecuencia de los signos de complicación en general y por edades.

\section{Exámenes de laboratorio}

En la figura 1 se muestra la tendencia de las plaquetas, los leucocitos y el hematocrito durante la evolución. El 44,3\% de los pacientes presentó niveles de plaquetas menor o igual a 50.000 al ingreso, que ascendió a 74,7\% en algún momento de la estancia. La trombocitopenia intensa fue más común en los adultos que en los niños (81,3 Vs. $54,88 \%, R R=1,48, I C 95 \% 1,21-1,82, p<0,001)$. Por otro lado, $35,4 \%$ de los pacientes presentaron un viraje del hematocrito mayor o igual a $20 \% ; 42,1 \%$, entre 10 y $20 \%$, y $22,6 \%$, de menos del $10 \%$. La hemoconcentración mayor o igual a $20 \%$ fue más común en los niños respecto a los adultos (45,12 Vs. 32,11\%, RR=1,41, IC95\% 1,04-1,9, $\mathrm{p}=0,045)$.

Se midieron transaminasas en 159 pacientes $(48,5 \%)$, y se encontró un incremento de 4,15

Cuadro 2. Signos vitales según día de enfermedad.

\begin{tabular}{|c|c|c|c|c|c|c|c|c|}
\hline Día & & 4 & 5 & 6 & 7 & 8 & 9 & $\mathbf{p}^{*}$ \\
\hline \multirow[t]{4}{*}{ PA } & Adultos & S: $110(80-155)$ & S: 108 (70-160) & S: 106,5 (70-180) & S: $110(60-160)$ & S: 110 (80-170) & S: $110(80-170)$ & $\begin{array}{l}\text { S: } 0,0001 \\
\text { D: } 0,0901\end{array}$ \\
\hline & & D: $70(40-110)$ & D: $70(40-100)$ & D: 70 (40-101) & D: $70(30-110)$ & D: 70 (40-110) & D: 70 (50-100) & $\mathrm{M}: 0,0047$ \\
\hline & Niños & S: $100(80-114)$ & S: 99 (48-140) & S: 94,5 (70-120) & S: 91 (70-123) & S: 90 (80-120) & S: 90 (61-130) & $\begin{array}{l}\text { S: } 0,7708 \\
\text { D: } 0,2307\end{array}$ \\
\hline & & D: 60 (50-86) & D: 60 (20-98) & D: 60 (40-80) & D: 60 (40-100) & D: 60 (44-80) & D: 60 (34-90) & M: 0,2069 \\
\hline \multicolumn{2}{|c|}{ Hipotensión ${ }^{\dagger}$} & 1,09 Vs. 46,15 & 1,24 Vs. 50 & 0,51 Vs. 59,72 & 0,52 Vs. 58,9 & 0,65 Vs. 62,26 & 0 Vs. 53,66 & \\
\hline & $\mathrm{p}^{\ddagger}$ & $<0,001$ & $<0,001$ & $<0,001$ & $<0,001$ & $<0,001$ & $<0,001$ & \\
\hline \multirow[t]{2}{*}{$\mathrm{FC}$} & Adultos & $84(48-130)$ & $80(47-120)$ & $80(60-104)$ & $80(60-114)$ & $80(52-105)$ & $80(60-92)$ & 0,0001 \\
\hline & Niños & $96(54-130)$ & $100(53-149)$ & $98,5(71-170)$ & $96(68-174)$ & $94,5(68-170)$ & $94,5(63-173)$ & 0,3172 \\
\hline \multicolumn{2}{|c|}{ Taquicardia§ } & 9,78 Vs. 11,43 & 6,83 Vs. 14,49 & 0,51 Vs. 14,77 & 0,51 Vs. 7,87 & 0,66 Vs. 10 & 0 Vs. 8,7 & \\
\hline & $\mathrm{p}^{\ddagger}$ & 0,752 & 0,081 & $<0,001$ & 0,001 & 0,002 & 0,022 & \\
\hline \multirow[t]{2}{*}{$\mathrm{RR}$} & Adultos & $20(12-32)$ & $20(10-36)$ & $20(14-30)$ & $20(14-36)$ & $20(14-27)$ & $20(16-24)$ & 0,2485 \\
\hline & Niños & $22(18-36)$ & $22,5(14-47)$ & $24(16-64)$ & $24(12-68)$ & $24(16-48)$ & $24(14-48)$ & 0,0712 \\
\hline \multicolumn{2}{|c|}{ Taquipnea ${ }^{\S}$} & 24,44 Vs. 40 & 24,38 Vs. 35,29 & 23,98 Vs. 43,18 & 26,9 Vs. 47,19 & 15,79 v. 44,93 & 14,08 v. 40,43 & \\
\hline & $\mathrm{p}^{\ddagger}$ & 0,123 & 0,106 & 0,002 & 0,001 & $<0,001$ & 0,002 & \\
\hline \multirow[t]{2}{*}{$\mathrm{T}$} & Adultos & $37(35,8-39,5)$ & $37(34-40,5)$ & $36,7(35,8-38,8)$ & $36,6(35,4-39)$ & $36,6(35,9-39)$ & $36,7(35,8-39)$ & 0,0026 \\
\hline & Niños & $37(35,5-39,4)$ & $36,6(35-39,5)$ & $36,75(36-39)$ & $36,6(35,6-39)$ & $36,6(35-38,5)$ & $36,5(36-39,4)$ & 0,0492 \\
\hline \multirow[t]{2}{*}{ Fiebrell } & & 27,59 Vs. 28,57 & 13,33 v. 15,38 & 6,49 Vs. 8,33 & 2,69 v. 6,98 & 2,74 Vs. 4,48 & 2,9 v. 10,87 & \\
\hline & $\mathrm{p}^{\ddagger}$ & 1 & 0,674 & 0,612 & 0,107 & 0,681 & 0,114 & \\
\hline
\end{tabular}

PA: presión arterial ( $\mathrm{mm} \mathrm{Hg}$ ), S: sistólica, D: diastólica, M: media, FC: frecuencia cardiaca (latidos por minuto), FR: frecuencia respiratoria (ciclos por minuto), T: temperatura (grados Celsius); datos expresados como mediana (rango)

Para los signos vitales se definió adulto como edad mayor o igual a 18.

* Comparación entre días (prueba de Kruskal Wallis para variables de distribución no normal)

† Hipotensión: PA media menor a $60 \mathrm{~mm} \mathrm{Hg}$ en adultos y PA sistólica menor al percentil 5 para la edad en niños

¥ Comparación adultos Vs. niños de la proporción de pacientes con hipotensión, taquicardia, taquipnea o fiebre

$\S$ Se definieron taquicardia y taquipnea como $\mathrm{FC}>100$ y $\mathrm{FR}>20$, respectivamente para adultos, así como parámetros superiores al percentil 95 para la edad en niños.

\| Temperatura $\geq 38^{\circ} \mathrm{C}$ 
Cuadro 3. Signos de alarma y de complicación durante la estancia hospitalaria.

\begin{tabular}{|c|c|c|c|c|}
\hline & Adultos & Niños * & Total & p \\
\hline Ascitis & $7 \quad(2,85 \%)$ & $29(35,37 \%)$ & 36 (10,98\%) & $<0,001$ \\
\hline Derrame & $15 \quad(6,1 \%)$ & $19(23,17 \%)$ & $34 \quad(10,37 \%)$ & $<0,001$ \\
\hline Sangrado & $188(76,42 \%)$ & $53(64,63 \%)$ & $241 \quad(73,48 \%)$ & 0,043 \\
\hline Vómito & $56(22,76 \%)$ & $17(20,73 \%)$ & $73(22,26 \%)$ & 0,761 \\
\hline Dolor abdominal & $200 \quad(81,3 \%)$ & $58(70,73 \%)$ & $258 \quad(78,66 \%)$ & 0,061 \\
\hline Síntomas autonómicos & $13 \quad(5,28 \%)$ & $4 \quad(4,88 \%)$ & $17 \quad(5,18 \%)$ & 1 \\
\hline
\end{tabular}

* Para complicaciones se consideró niño como menor de 13 años
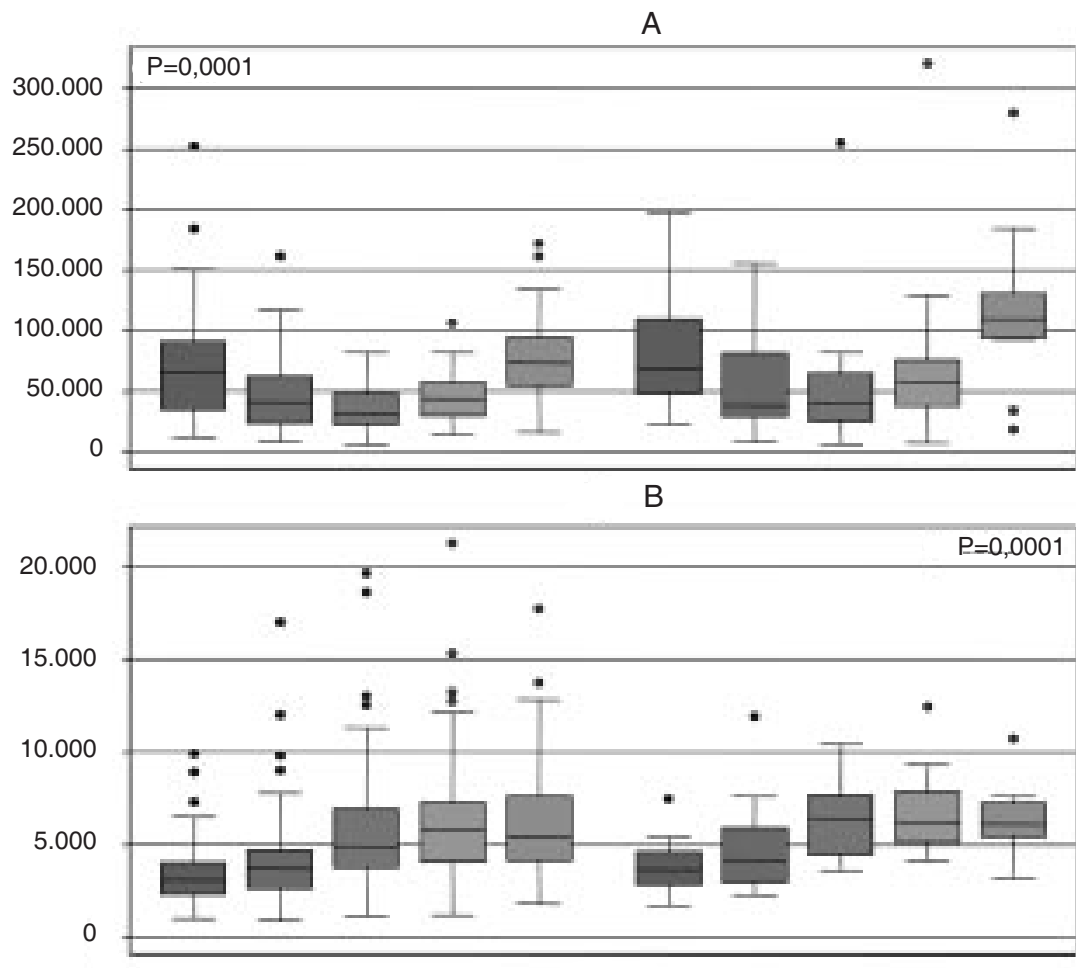

C

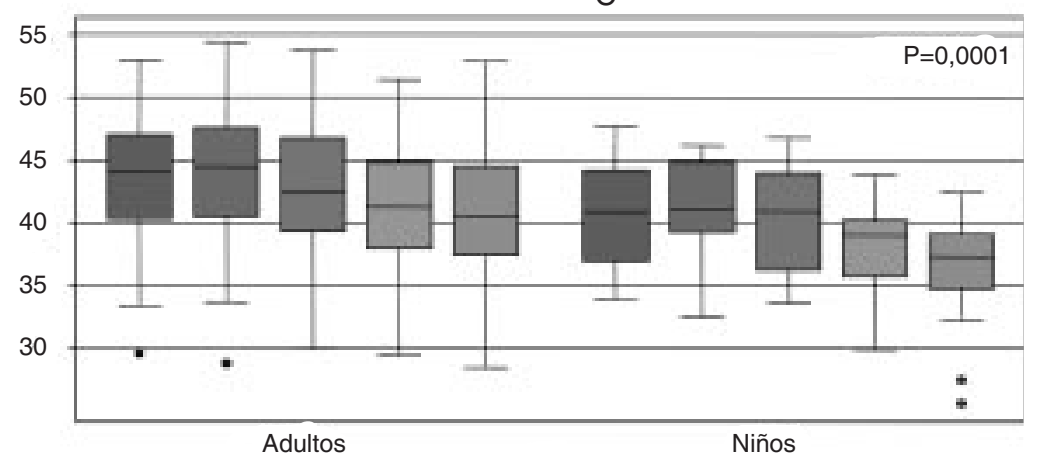

Figura 1. Historia natural de los parámetros hematológicos entre los días 4 y 8 de enfermedad según grupos de edad. A: plaquetas, B: leucocitos, C: hematocrito. P calculada para la comparación entre días. 
$(0,39-24,86)$ y $2,11(0,17-15,66)$ veces los valores de referencia para las transaminasas glutámicooxalacética y glutámico-pirúvica, respectivamente. Los pacientes con IgM positiva presentaban una elevación mayor de dichas enzimas que aquéllos con IgM negativa $(187$ [18-1051,6] Vs. 65 [16-132], $\mathrm{p}=0,0048$ y 122 [13-755] Vs. 55,4 [18133], $p=0,0235$, respectivamente), aunque no se encontraron diferencias significativas al comparar los pacientes con dengue hemorrágico y sin él.

Se practicaron 117 exámenes de orina a 98 pacientes, de los cuales, $52(44,4 \%)$ fueron normales. Se encontró hematuria en 37 (31,6\%) e infección en dos $(1,7 \%)$. Se realizaron 24 exámenes coprológicos en 23 pacientes. En 12 de ellos el examen fue normal, en 3 se detectaron hematíes y 6 fueron interpretados como infección. Para el diagnóstico diferencial se realizaron 13 exámenes de gota gruesa y 2 pruebas de antígeno de superficie para hepatitis $B$, todos ellos negativos. En el cuadro 4 se resumen los resultados de todas las pruebas de laboratorio realizadas.

\section{Imágenes diagnósticas}

Se practicaron 42 ecografías abdominales con barrido de bases pulmonares a 40 pacientes $(12,2 \%)$; la ascitis fue el hallazgo más frecuente $(80 \%)$, seguido por edema de la pared de la vesícula biliar $(60 \%)$, derrame pleural $(57,5 \%)$, hepatomegalia (17,5\%), esplenomegalia $(2,5 \%)$ e inflamación del hígado (2,5\%). El examen fue normal en $17,5 \%$. Se hizo ecografía hepatobiliar a 6 pacientes, 4 de las cuales fueron normales; los hallazgos anormales fueron hepatomegalia en 2 , colelitiasis y edema de pared de vesícula biliar en 1 . Se realizaron 17 radiografías de tórax, 3 de ellas normales. Se encontró derrame pleural en 11 , infiltrados en 3 , consolidación neumónica en una y signos de sobrecarga en una.

\section{Tratamiento hospitalario}

En el cuadro 5 se resumen las necesidades de líquidos endovenosos según edad y día de evolución. Se utilizaron cristaloides de manera exclusiva, excepto por coloides en un paciente con choque resistente al tratamiento. Además de

Cuadro 4. Resultados de los exámenes de laboratorio.

\begin{tabular}{|c|c|c|c|c|}
\hline Examen & $\mathbf{n}$ & Mediana & Rango & \% Alteración \\
\hline \multicolumn{5}{|c|}{ Pruebas de coagulación } \\
\hline TP & 84 & 13,8 & $10-27$ & 21,43 \\
\hline INR & 52 & 1,21 & $0,96-2,44$ & 51,92 \\
\hline TPT & 81 & 41,7 & $26-120$ & 80,25 \\
\hline \multicolumn{5}{|l|}{ Electrolitos } \\
\hline Sodio & 22 & 138,1 & $116-165$ & 18,18 \\
\hline Cloro & 22 & 107,5 & $91,9-117$ & 4,55 \\
\hline Potasio & 22 & 3,66 & $2,04-4,74$ & 31,82 \\
\hline \multicolumn{5}{|l|}{ Otros } \\
\hline Albúmina & 10 & 2,68 & $2-3,56$ & 80 \\
\hline Bilirrubina directa & 38 & 0,59 & $0,12-4,5$ & 68,42 \\
\hline Bilirrubina indirecta & 37 & 0,4 & $0,02-3,97$ & 37,84 \\
\hline Bilirrubina total & 38 & 0,9 & $0,21-6,05$ & 42,11 \\
\hline BUN & 25 & 10 & $5,1-27,5$ & 24 \\
\hline Creatinina & 38 & 0,87 & $0,3-1,77$ & 28,95 \\
\hline Fosfatasa alcalina & 30 & 233,5 & $31,9-1225$ & 70 \\
\hline LDH & 8 & 578 & $308,3-1.440$ & 100 \\
\hline PCR cuantitativa & 25 & 12 & $2,5-192$ & 88 \\
\hline Proteínas totales & 7 & 4,5 & $3,8-5,6$ & 100 \\
\hline VSG & 11 & 10 & $2-42$ & 63,64 \\
\hline
\end{tabular}

TP, tiempo de protrombina; INR international normalized ratio; TPT, tiempo parcial de tromboplastina; BUN, nitrógeno ureico; $\mathrm{LDH}$, lactato deshidrogenasa; PCR, proteína C reactiva; VSG velocidad de sedimentación globular. 
Cuadro 5. Necesidad de líquidos endovenosos según día de enfermedad.

\begin{tabular}{|c|c|c|c|c|c|c|}
\hline \multirow[b]{2}{*}{ Día } & \multicolumn{2}{|c|}{ Adultos } & \multirow[b]{2}{*}{$\mathrm{DE}$} & \multicolumn{3}{|c|}{ Niños } \\
\hline & $\mathrm{n}$ & Promedio* & & $\mathrm{n}$ & Promedio & DE \\
\hline 4 & 91 & 2500 & 1284,523 & 21 & 1523,81 & 828,7972 \\
\hline 5 & 173 & 2696,532 & 1447,367 & 44 & 1886,364 & 1027,888 \\
\hline 6 & 216 & 2916,667 & 1224,745 & 56 & 1732,143 & 1086,965 \\
\hline 7 & 207 & 2724,638 & 1356,044 & 59 & 1500 & 1078,792 \\
\hline 8 & 151 & 2039,735 & 1248,363 & 44 & 1488,636 & 979,3723 \\
\hline 9 & 67 & 1955,224 & 1491,723 & 26 & 1192,308 & 749,3587 \\
\hline
\end{tabular}

* Expresado en centímetros cúbicos de cristaloides por día.

Comparación entre días: $p<0,0001$ para adultos, $p=0,06$ para niños (menores de 13 años)

la hidratación, los medicamentos más frecuentes según la indicación fueron:

- Analgésicos: acetaminofén, 72\%; diclofenaco, $11,9 \%$; dipirona, 10,4\%; hioscina, 5,8\%, y tramadol, 2,7\%.

- Antimicrobianos: 13 pacientes $(3,6 \%)$.

- Soporte crítico: furosemida, $4 \%$; hidrocortisona, 2,4\%; vitamina $\mathrm{K}, 2,1 \%$, y ácido tranexámico, $1,3 \%$.

- Manejo sintomático y soporte básico: omeprazol, $52,1 \%$; metoclopramida, 50,3\%; sucralfate, $32,6 \%$; ranitidina, $21,3 \%$; loratadina, $15,6 \%$, y oxígeno, $6,1 \%$.

- Transfusiones: 15 pacientes $(4,6 \%)$ recibieron transfusiones de plaquetas, $8(2,4 \%)$ de plasma fresco congelado, $3(0,9 \%)$ de glóbulos rojos empaquetados y $1(0,3 \%)$ de crioprecipitados. Este tratamiento, en particular, fue casi exclusivo de los pacientes con dengue hemorrágico $(80,95$ Vs. $19,05 \%$ en dengue clásico; $R R=7,77, \operatorname{IC95\% ~2,68-22,54,~}$ $\mathrm{p}<0,0001$ ).

\section{Complicaciones}

Se consideraron complicaciones clínicas el sangrado mayor (hematemesis o melenas, $5,2 \%$ ), los signos clínicos de extravasación plasmática (ascitis o derrame, 14,6\%), la dificultad respiratoria $(6,7 \%)$ y la hipotensión $(24,7 \%)$. Ocurrió un caso fatal con sangrado masivo, coagulación intravascular diseminada y choque resistente al tratamiento, para una mortalidad de $0,3 \%$.
Otras condiciones diagnosticadas concomitantemente con el dengue fueron neumonía bacteriana en dos pacientes (uno de los cuales desarrolló sepsis), hepatopatía clínica en dos casos e insuficiencia renal en uno. Dos pacientes embarazadas no presentaron complicaciones obstétricas durante la estancia hospitalaria, aunque en una de ellas el dengue cursó con un episodio de hiperemesis gravídica.

\section{Clasificación de la OMS}

Se cumplieron los criterios clínicos para caso probable de dengue en 299 pacientes $(91,2 \%)$, de los cuales, 151 tuvieron IgM positiva, 34 negativa y 114 sin dato. Las figuras 2 y 3 muestran el diagnóstico de dengue hemorrágico en todos los pacientes del estudio. La distribución del dengue hemorrágico fue de 1, 113, 1 y 1 para los grados I a IV, respectivamente. De los 116 pacientes con dengue hemorrágico, 44 presentaban la complicación desde el ingreso.

Usando las definiciones tradicionales de la OMS, se encontró que los pacientes con dengue hemorrágico presentaban un promedio de edad menor $(20,06$ Vs. $25,67, p=0,0054)$ y una estancia hospitalaria un día mayor (mediana 4 contra 3 días, $p=0,0001$ ). También, se evidenció una ligera diferencia por sexo de la presentación de esta forma grave, pero no fue significativa $(40 \%$ en mujeres Vs. 30,7\% en hombres, $p=0,084)$.

Se probaron dos alternativas más flexibles de la definición de la OMS: al incluir un hematocrito superior a $50 \%$ como signo de fuga vascular, los 


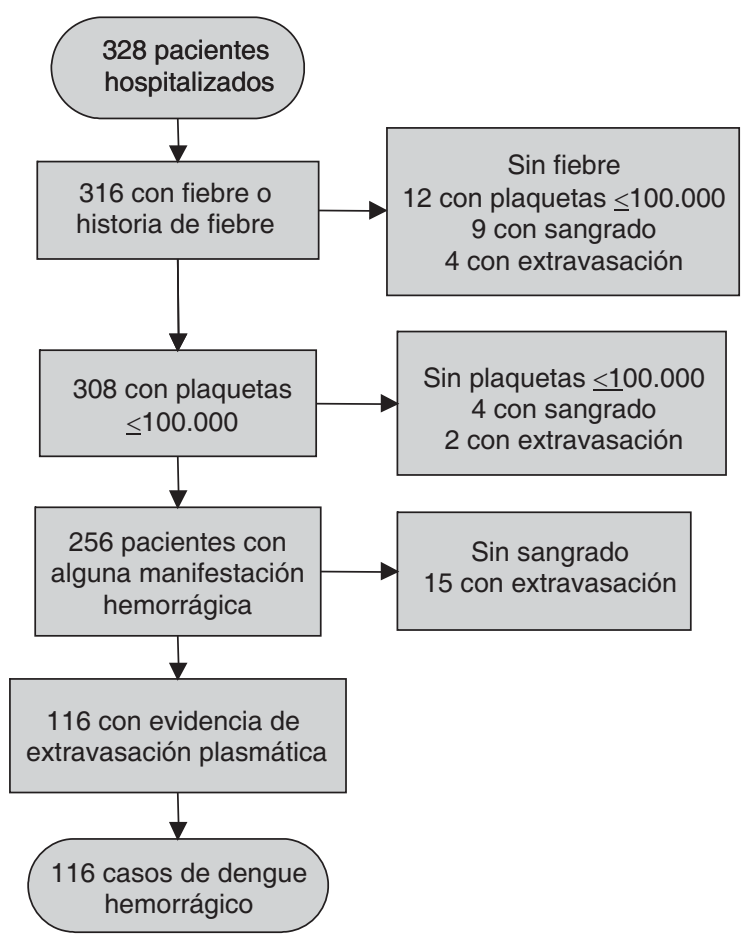

Figura 2. Flujograma que muestra el diagnóstico de dengue hemorrágico en los pacientes del estudio usando los criterios de la OMS.

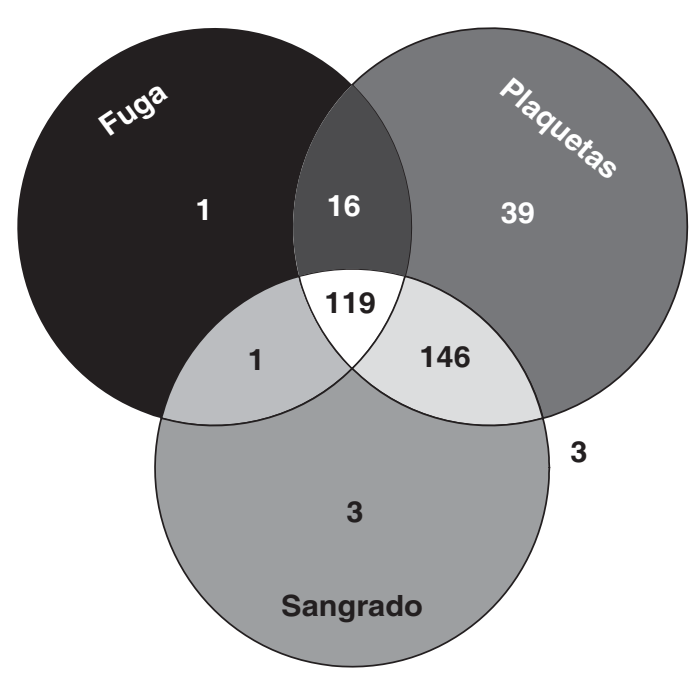

Figura 3. Diagrama de Venn que muestra el comportamiento de los criterios para dengue hemorrágico. Para esta distribución se asume fiebre en todos los pacientes del estudio, pese a que no se pudo confirmar en diez de los casos por vacíos en la historia clínica. casos de dengue hemorrágico aumentaron a 123; y si, además, se redefine el colapso circulatorio como presión de pulso estrecha o hipotensión en compañía de signos de choque (pulso débil, hipoperfusión o frialdad), los casos de síndrome de choque por dengue ascienden a 7.

\section{Pronóstico hospitalario}

Para cada uno de los desenlaces de mal pronóstico hospitalario se evaluaron diferentes factores de riesgo presentados al ingreso, todos ajustados por edad, sexo y día de evolución de la enfermedad. No se encontraron diferencias significativas con dengue hemorrágico (incidencia de 25,7\%). El riesgo de presentar hipotensión (13,3\%) fue mayor en los pacientes que refirieron cefalea $(R R=1,95$, IC95\% 1,35-2,82, $\mathrm{p}<0,001)$, dolor abdominal ( $R R=2,88, I C 95 \% 1,22-6,82, p=0,016)$ y sangrado $(R R=2,38, I C 95 \% 1,6-3,56, p<0,001) . L a$ hemoglobina al ingreso también se asoció con este desenlace, probablemente como signo indirecto de hemoconcentración (RR=1,19, IC95\% 1,12$1,27, p<0,001)$.

Con sangrado mayor (incidencia de $3,12 \%$ ), sólo se encontró asociada la astenia ( $R R=0,25, I C 95 \%$ $0,07-0,89, p=0,032$ ). En cuanto a la extravasación plasmática (criterios de la OMS, incidencia de $27,92 \%)$, se hallaron relacionadas la frecuencia respiratoria de ingreso ( $R R=1,07, I C 95 \% 1,04-1,09$, $p<0,001)$ y el recuento de linfocitos en el cuadro hemático inicial $(R R=0,75$ para cada incremento de 10 células en el diferencial, IC95\% 0,67-0,84, $\mathrm{p}<0,001)$.

En esta cohorte la incidencia de traslado a cuidados intensivos fue de 2,2\%. La hipotensión (RR=10,98, IC95\% 1,03-117,65, p=0,048) y la hemoconcentración (como variable continua, $R R=1,09$, IC95\% 1,008-1,17, p=0,03) influyeron en dicha decisión. En el cuadro 6 se listan los factores de riesgo asociados a dificultad respiratoria (incidencia de 5,85\%).

La edad mostró ser factor de riesgo independiente para el desarrollo de complicaciones: los menores de 13 años tuvieron mayor riesgo de presentar dificultad respiratoria (RR=3,59, IC95\% 1,3-9,9, $\mathrm{p}=0,014)$. Además, por cada año adicional de edad, la probabilidad de hipotensión hospitalaria 
Cuadro 6. Factores de riesgo al ingreso para el desarrollo de dificultad respiratoria.

\begin{tabular}{|c|c|c|c|c|c|c|}
\hline \multirow[b]{2}{*}{ Variable } & \multicolumn{3}{|c|}{ Análisis bivariado } & \multicolumn{3}{|c|}{ Posterior al ajuste * } \\
\hline & $\mathbf{R R}$ & IC95\% & $\mathbf{p}$ & $\mathbf{R R}$ & IC95\% & $\mathbf{p}$ \\
\hline Síntomas respiratorios $^{\dagger}$ & 4,07 & $1,49-11,12$ & 0,0223 & 5,26 & $1,37-20,23$ & 0,016 \\
\hline Vómito & 3,5 & $1,19-10,33$ & 0,0171 & 2,47 & $0,84-7,29$ & 0,102 \\
\hline Sangrado (signo) & 2,41 & $0,99-5,83$ & 0,0514 & 2,36 & $1,002-5,54$ & 0,049 \\
\hline Hemoconcentración †‡ & & & 0,0001 & 1,14 & 1,07 - 1,22 & $<0,001$ \\
\hline Leucocitos ${ }^{\dagger \ddagger \S}$ & & & 0,0008 & 1,02 & $1,003-1,04$ & 0,02 \\
\hline Presión arterial sistólica $¥ \|$ & & & 0,049 & 0,73 & $0,59-0,91$ & 0,005 \\
\hline Frecuencia respiratoria $\ddagger$ & & & 0,038 & 1,09 & $1,02-1,16$ & 0,038 \\
\hline Frecuencia cardiaca $\ddagger \|$ & & & 0,0005 & 1,03 & $1,005-1,05$ & 0,016 \\
\hline
\end{tabular}

* Ajustado por edad, sexo y día de evolución al ingreso

† Para esta variable se calculó OR en lugar de RR en el ajuste por problemas de convergencia.

¥ Tomada como variable continua

$\S$ Riesgo calculado para cada incremento de 100 unidades en el valor de la variable

|| Riesgo calculado para cada incremento de 10 unidades en el valor de la variable

disminuyó en $18 \%(\mathrm{OR}=0,82$, IC95\% 0,76-0,88, $p<0,001)$.

\section{Diagnóstico serológico}

Se pudo obtener confirmación serológica de 193 pacientes (58,8\%). Al comparar los grupos de individuos con IgM positiva, negativa o ausente, se encontraron diferencias significativas en el tiempo de evolución de los síntomas en la enfermedad actual (un día menor en los pacientes con IgM negativa, $p=0,0179$ ), la proporción de pacientes con dengue hemorrágico según la OMS $(41,51 \%$, $38,24 \%$ y $27,41 \%$, respectivamente, $p=0,038)$ y la presencia de cualquier sangrado $(79,87 \%$, $85,29 \%$ y $62,96 \%$, respectivamente, $p=0,001$ ), aunque no en los desenlaces de gravedad evaluados ni en los valores de plaquetas y hematocrito.

\section{Discusión}

De manera general, la gravedad en este estudio fue menor en comparación con otros trabajos realizados en población hospitalaria en Colombia. Actualmente no hay consenso en cuanto al papel del sexo en el pronóstico del dengue y, aunque encontramos que las mujeres presentaban con mayor frecuencia los signos de alarma, esto no se tradujo en un aumento de la gravedad.

Por el contrario, la edad continúa siendo un factor determinante: los menores de 13 años presentaron un cuadro clínico atípico, con menor frecuencia de sangrado y trombocitopenia pro- funda en comparación con adolescentes y adultos, pero con mayor riesgo de complicaciones hospitalarias, incluso dengue hemorrágico. Cabe resaltar que para este último desenlace los pacientes entre 20 y 30 años siguieron en frecuencia, lo cual sugiere que en Colombia también puede presentarse el fenómeno de desplazamiento de la gravedad hacia grupos de mayor edad.

El patrón de enfermedad obtenido en este estudio concuerda con el descrito en América Latina (19$23,29,30)$. Sin embargo, nuestra población se asemeja más a la descrita en estudios internacionales que en los locales, en la menor frecuencia de hemorragia gastrointestinal $(11,12,16)$ y en un mayor compromiso no diferencial en los tiempos de coagulación (31).

Encontramos, asimismo, dificultades para clasificar nuestros pacientes de acuerdo con la definición de la OMS $(21,22,29,30)$. Un porcentaje importante de pacientes graves no lograron reunir todos los criterios de la OMS para dengue hemorrágico. Igualmente, 17 de 116 pacientes con dengue hemorrágico no desarrollaron hemoconcentración mayor o igual a $20 \%$, de los cuales 5 presentaron una ecografía positiva como único signo de extravasación, indicando su utilidad diagnóstica $(32,33)$.

Dos limitaciones de este estudio fueron la falta de confirmación serológica en un $41,2 \%$ de los 
casos estudiados y el uso de historias clínicas como fuente de información. Asimismo, varias características del trabajo limitan la población a la cual pueden aplicarse estos resultados. Primero, la proporción de niños con dengue hemorrágico puede ser distinta a la real dado que Bucaramanga cuenta con un centro especializado en población infantil al que se remiten muchos de los casos. Segundo, la acentuada diferencia en el perfil clínico del presente estudio, realizado en un periodo interepidémico, comparado con el encontrado en los demás trabajos colombianos, hechos durante brotes o epidemias (19-23), podría explicar porqué encontramos una baja incidencia de complicaciones y una mínima mortalidad que no serían atribuibles únicamente a la calidad de la atención prestada. Finalmente, dadas las desigualdades del sistema de salud en nuestro país, los hallazgos encontrados no podrán generalizarse adecuadamente a la población que no pertenece al régimen contributivo.

Muchos pacientes hospitalizados presentaron estabilidad hemodinámica durante toda su estancia, asintomáticos o con molestias menores, sin sangrado o sólo con petequias, sin signos de extravasación y con trombocitopenia como único criterio de seguimiento. Este estudio se suma a otros que consideran posible seleccionar a estos pacientes con bajo riesgo de complicación (3436), quienes serían potenciales candidatos a programas de atención diferentes a la hospitalización, lo cual reduciría el riesgo de infecciones hospitalarias y liberaría valiosos recursos para la atención en salud. La anterior estrategia será posible una vez contemos con marcadores pronósticos válidos y reproducibles de la gravedad de la enfermedad que nos permitan identificar tempranamente a dichos pacientes, cuya atención oportuna impactaría favorablemente la morbilidad y mortalidad del dengue en nuestro país.

En conclusión, la edad continúa siendo el factor predominante en la gravedad hospitalaria del dengue. Independientemente de ella, un grupo de signos, síntomas y hallazgos de laboratorio al momento del ingreso permite predecir la aparición de complicaciones. Esto puede ser de utilidad para personalizar el tratamiento y optimizar los recursos asistenciales en las áreas endémicas o de manera general en periodos epidémicos.

\section{Agradecimientos}

Gracias de manera muy especial a todo el personal de la Clínica Chicamocha por hacer posible la realización de este trabajo y a Alba Nury Ramírez de la Secretaría de Salud de Santander por su colaboración en la obtención de los datos de vigilancia epidemiológica.

\section{Conflicto de intereses}

Los autores declaramos que no existe conflicto de intereses.

\section{Financiación}

Este trabajo fue financiado por Colciencias, la Clínica Chicamocha y la Universidad Industrial de Santander bajo el marco del convenio "Jóvenes Investigadores e Innovadores" modalidad empresarial, código CV 130-2006.

\section{Referencias}

1. World Health Organization. Dengue and dengue haemorrhagic fever. Geneva: WHO; 2008. [Fecha de consulta: 18 de agosto de 2008]. Disponible en: http:// www.who.int/mediacentre/factsheets/fs117/en/

2. Kurane I. Dengue hemorrhagic fever with special emphasis on immunopathogenesis. Comp Immunol Microbiol Infect Dis. 2007;30:329-40.

3. World Health Organization. Dengue and dengue haemorrhagic fever. In: WHO Report on Global Surveillance of Epidemic-prone Infectious Diseases. First edition. Geneva: WHO; 2000. p. 75-8. [Fecha de consulta: 30 de enero de 2008]. Disponible en: http:// www.who.int/entity/csr/resources/publications/surveillance/dengue.pdf

4. Pan American Health Organization. Number of reported cases of dengue and dengue hemorrhagic fever (DHF), Region of the Americas (by country and subregion). Washington D.C.: PAHO; 2008. [Consultada: 5 de marzo de 2008]. Disponible en: http:/ /www.paho.org/English/AD/DPC/CD/dengue-cases2008.pdf.

5. Pan American Health Organization. The history of dengue and dengue hemorrhagic fever (DHF) in the Region of the Americas, 1635-2001. Washington D.C.: PAHO; 2001. [Fecha de consulta: 30 de enero de 2008]. Disponible en: http://www.ops-oms.org/English/AD/ DPC/CD/dengue_history.htm

6. Instituto Nacional de Salud. Casos totales en la semana epidemiológica 52 y acumulados del año. 
Bogotá D.C.: INS; 2006. [Fecha de consulta: 18 de agosto de 2008]. Disponible en: http://www.ins.gov.co/ pdf/vcsp/Tablas/2006/2006_semana_52.pdf

7. Instituto Nacional de Salud. Casos totales en la semana epidemiológica 50 y acumulados del año. Bogotá D.C.: INS; 2007. [Fecha de consulta: 18 de agosto de 2008]. Disponible en: http://www.ins.gov.co/ pdf/vcsp/Tablas/2007/2007_semana_50.pdf

8. World Health Organization. Guidelines for treatment of dengue fever/dengue haemorrhagic fever in small hospitals. Geneva: WHO; 1999. p. 28. [Fecha de consulta: 30 de enero de 2008]. Disponible en: http:// www.searo.who.int/LinkFiles/Dengue_Guidelinedengue.pdf

9. Méndez A, Bárcenas C, González G, Villar LA, Sepúlveda JW, Rey JJ, et al. Protocolo para la atención del dengue y dengue hemorrágico en el municipio de Bucaramanga. Bucaramanga: Secretaría de Salud y del Ambiente; 2003. p. 48. [Fecha de consulta: 30 de enero de 2008]. Disponible en: http://www. bucaramanga. gov. co/docs/Protocolo\% 20manejo\% 20dengue.pdf

10. Martínez-Vega RA, Díaz-Quijano FA, Villar-Centeno LA. Low concordance between early clinical suspicion of dengue and its serological confirmation. Rev Med Chil. 2006;134:1153-60.

11. Khan NA, Azhar El, El-Fiky S, Madani HH, Abuljadial MA, Ashshi AM, et al. Clinical profile and outcome of hospitalized patients during first outbreak of dengue in Makkah, Saudi Arabia. Acta Trop. 2008;105:39-44.

12. Malavige GN, Velathanthiri VG, Wijewickrama ES, Fernando S, Jayaratne SD, Aaskov J, et al. Patterns of disease among adults hospitalized with dengue infections. QJM. 2006;99:299-305.

13. Kularatne SA, Gawarammana IB, Kumarasiri PR. Epidemiology, clinical features, laboratory investigations and early diagnosis of dengue fever in adults: a descriptive study in Sri Lanka. Southeast Asian J Trop Med Public Health. 2005;36:686-92.

14. Shah I, Deshpande GC, Tardeja PN. Outbreak of dengue in Mumbai and predictive markers for dengue shock syndrome. J Trop Pediatr. 2004;50:301-5.

15. Murgue B, Deparis X, Chungue E, Cassar O, Roche C. Dengue: an evaluation of dengue severity in French Polynesia based on an analysis of 403 laboratory-confirmed cases. Trop Med Int Health. 1999;4:765-73.

16. Tai DY, Chee YC, Chan KW. The natural history of dengue illness based on a study of hospitalised patients in Singapore. Singapore Med J. 1999;40:238-42.

17. Malavige GN, Ranatunga PK, Velathanthiri VG, Fernando S, Karunatilaka DH, Aaskov J, et al. Patterns of disease in Sri Lankan dengue patients. Arch Dis Child. 2006;91:396-400.

18. Lee VJ, Lye DC, Sun Y, Fernandez G, Ong A, Leo YS. Predictive value of simple clinical and laboratory variables for dengue hemorrhagic fever in adults. $\mathrm{J}$ Clin Virol. 2008;42:34-9.

19. Rosso F, Restrepo M, Alzate A, Muñoz J, Moreno C. Dengue hemorrágico en el Hospital Universitario del Valle, 1990-1992. Colomb Med. 1994;25:10-4.

20. Salgado D, Rodríguez A, Vega R. Dengue hemorrágico: emergencia pediátrica en el Huila. Pediatría (Bogotá). 1999;34:78-83.

21. Méndez A, González G. Dengue hemorrágico en niños: diez años de experiencia clínica. Biomédica. 2003;23:180-93.

22. Arboleda M, Campuzano M, Restrepo BN, Cartagena G. Caracterización clínica de los casos de dengue hospitalizados en la E.S.E. "Antonio Roldán Betancur" Apartadó, Antioquia, 2000. Biomédica. 2006;26:286-94.

23. Salgado DM, Rodríguez JA, Garzón M, Cifuentes G, Ibarra M, Vega MR, et al. Clinical and epidemiological characterisation of dengue haemorrhagic fever in Neiva, Colombia, 2004. Rev Salud Pública (Bogotá). 2007;9:53-63.

24. Guha-Sapir D, Schimmer B. Dengue fever: new paradigms for a changing epidemiology. Emerg Themes Epidemiol. 2005;2:1.

25. Park MK, Menard SW, Schoolfield J. Oscillometric blood pressure standards for children. Pediatr Cardiol. 2005;26:601-7.

26. World Health Organization. Clinical diagnosis. In: Dengue haemorrhagic fever: diagnosis, treatment, prevention and control. Second edition. Geneva: WHO; 1997. p. 12-23. [Fecha de consulta: 30 de enero de 2008]. Disponible en: http://www.who.int/entity/csr/resources/publications/dengue/012-23.pdf

27. McPhee SJ, Papadakis MA, Tierney LM. Appendix, therapeutic drug monitoring \& laboratory reference ranges. In: Current medical diagnosis \& treatment. 46th edition. New York: McGraw-Hill; 2007. p. 1768-77.

28. Hay W, Levin M, Sondheimer J, Deterding R. Chemistry \& hematology reference intervals. In: Current diagnosis \& treatment in pediatrics. 18th edition. New York: McGraw-Hill; 2007. p. 1294-306.

29. Deen JL, Harris E, Wills B, Balmaseda A, Hammond SN, Rocha C, et al. The WHO dengue classification and case definitions: time for a reassessment. Lancet. 2006;368:170-3.

30. Balmaseda A, Hammond SN, Perez MA, Cuadra R, Solano S, Rocha J, et al. Short report: assessment of the World Health Organization scheme for classification of dengue severity in Nicaragua. Am J Trop Med Hyg. 2005;73:1059-62.

31. Avila-Aguero ML, Avila-Aguero CR, Um SL, Soriano-Fallas A, Canas-Coto A, Yan SB. Systemic host inflammatory and coagulation response in the dengue virus primo-infection. Cytokine. 2004;27:173-9. 
32. Venkata Sai PM, Dev B, Krishnan R. Role of ultrasound in dengue fever. Br J Radiol. 2005;78:416-8.

33. Srikiatkhachorn A, Krautrachue A, Ratanaprakarn W, Wongtapradit L, Nithipanya N, Kalayanarooj S, et al. Natural history of plasma leakage in dengue hemorrhagic fever: a serial ultrasonographic study. Pediatr Infect Dis J. 2007;26:283-90.

34. Díaz FA, Martínez RA, Villar LA. Criterios clínicos para diagnosticar el dengue en los primeros días de enfermedad. Biomédica. 2006;26:22-30.
35. Lee VJ, Lye DC, Sun Y, Fernandez G, Ong A, Leo YS. Predictive value of simple clinical and laboratory variables for dengue hemorrhagic fever in adults. J Clin Virol. 2008;42:34-9.

36. Tanner L, Schreiber M, Low JG, Ong A, Tolfvensta T, Lai YL et al. Decision tree algorithms predict the diagnosis and outcome of dengue fever in the early phase of illness. PLoS Negl Trop Dis. 2008;2:e196. 